\title{
The Effect of Murraya koenigii Extract on Therapeutic Efficacy of Amlodipine in Rats: Possible Drug-Herb Interaction
}

\author{
Kalyani H Barve*, Ginpreet Kaur, Aadesh Kumar and Saloni Daftardar
}

SPP-School of Pharmacy and Technology Management, SVKM's NMIMS, Mumbai, India

*Corresponding author: Kalyani Barve, SPP-SPTM, SVKM's NMIMS, 5th Floor Mithibai college building, V.L.Mehta Road, Vile Parle (W), Mumbai-400056, India, Tel: 9920467646; Fax 91-22-26185422; E-mail: barve.kalyani@gmail.com

Rec date: Aug 04, 2014, Acc date: Nov 14, 2014, Pub date: Nov 21, 2014

Copyright: ( 2015 Kalyani HB, et al. This is an open-access article distributed under the terms of the Creative Commons Attribution License, which permits unrestricted use, distribution, and reproduction in any medium, provided the original author and source are credited.

\begin{abstract}
Introduction: Murraya koenigii is commonly available condiment having a wide spectrum of pharmacological activities. It has folklore use for treatment of hypertension and is reported to have ACE inhibitory activity. Therefore, the present study is proposed to identify the antihypertensive effect of the Murraya koenigii. The increasing use of herbal medicine has raised the concern of the potential interactions between the herbs and the conventional drugs. Hence we have further tried to investigate the interactions of this extract with amlodipine, which in turn has a narrow therapeutic index.
\end{abstract}

Method: The alcoholic extract of the leaves was prepared and subjected to acute toxicity studies as per the OECD guideline 423. The extract was evaluated for its antihypertensive effect on cadmium chloride induced hypertension in rats. The extract was also co administered with amlodipine in normal rats to study pharmacodynamic interactions.

Result: The extract was found to contain steroids, alkaloids, glycosides, flavonoids, phenolic compounds and carbohydrates. It was found to be safe at the dose of $2000 \mathrm{mg} / \mathrm{kg}$. The extract showed hypotensive effect at the dose of $250 \mathrm{mg} / \mathrm{kg}$ and an antihypertensive effect at the dose of $150 \mathrm{mg} / \mathrm{kg}$. The combination of extract with Amlodipine showed a profound hypotensive effect, suggesting a synergistic interaction between the extract and Amlodipine resulting in mortality.

Conclusion: To conclude with, the drug-herb interactions pose a serious threat to public and require immense research for their identification and investigation. When a combination therapy is used, factors such as a safe drug combination and dose adjustment should be considered by the physician especially with the drugs having narrow therapeutic indices.

Keywords: Curry leaves; Karri patta; Hypertension; Cadmium chloride; Diuresis; Amlodipine

\section{Introduction}

Murraya koenigii (Family: Rutaceae) commonly known as curry leaf or Kadi patta, is widely used in Indian cookery for flavouring foodstuffs, and is a treasure of beneficial components which include glycosides, carbazole alkaloids, koenigin, phenolic compounds, flavanoids, resin and volatile oil [1-5]. The plant has been used, since times ancient, in the traditional system of medicine [6]. Many pharmacological activities of like vasodialtory, antidiabetic, hypocholesterolemic, antiulcer, anti-diarrheal, phagocytic, analgesic ,antinociceptive and wound healing have already been reported. The curry leaves have been known to promote appetite and digestion and have been popularly used as stomachic, purgative, antiemetic, anti-inflammatory and carminative $[7,8]$, hence aptly known as cure leaf [9]. Since inflammation, oxidative stress and hypertension are interlinked with each other, $M$. koenigii might have a potential as an antihypertensive agent. Moreover, as per folklore, the leaf is said to possess antihypertensive effect. However, no scientific data exists to prove the same. It is essential to exploit Murraya koenigii for its antihypertensive effect in the field of medicinal and pharmaceutical sciences for culminating into some novel application.

The leaf is very widely used in every Indian household in cooking. Thus, a concomitant use of the leaf with conventional drug might lead to a substantial clinical herb-drug interaction. It is therefore extremely important to investigate the potential for pharmaco-dynamic interactions of $M$. koenigii and cardiovascular drugs with narrow therapeutic index like amlodipine.

Amlodipine is a long acting calcium channel blocker belonging to the class of di-hydro-pyridines which inhibits the movement of calcium ions into the vascular smooth muscles. Amlodipine is used in the treatment of hypertension and coronary artery disease. Adverse effects of amlodipine are peripheral edema, dizziness, palpitations and headache [10]. Due to its long half-life (about 33 hours), there are many incidences of potential interactions of Amlodipine in presence of various herbs [11]. Also, the drugs or herbs which inhibit cytochrome $\mathrm{P}_{450}$ CyP3A4, an enzyme that metabolizes Amlodipine, can lead to serious adverse interactions owing to the accumulation of the drug in body.

Thus, considering the aforementioned points and bearing in mind the potential of co administration of $M$. koenigii leaf in the form of food and amlodipine, this work was planned to investigate the possible 
Citation: Kalyani HB, Ginpreet K, Aadesh K, Saloni D (2015) The Effect of Murraya koenigii Extract on Therapeutic Efficacy of Amlodipine in Rats: Possible Drug-Herb Interaction. General Med 3: 1000153. doi:10.4172/2327-5146.1000153

Page 2 of 4

pharmaco-dynamic interactions in the form of effect on blood pressure following their simultaneous administration.

\section{Materials and Methods}

\section{Drugs and materials}

$M$. koenigii was purchased from the local market and authenticated by a botanist Dr. Ganesh Iyer, Ruia college, MUMBAI. All the chemicals and solvents (AR grade) were purchased from Sigma Aldrich India.

\begin{abstract}
Animals
Male swiss albino mice weighing 20-30 g were used for the acute toxicity studies. Antihypertensive studies were performed using adult Albino Wistar rats of either sex weighing 150-200 gm. The animals were obtained from Bharat Serum, Thane, and housed in cages for one week at standard laboratory conditions $\left(25^{\circ} \mathrm{C}+2^{\circ} \mathrm{C}\right)$, relative humidity $60 \pm 5 \%$ and 12 hours light and dark cycle. The diet was standard pellets and the water was given ad libitum. The studies were carried according to the CPCSEA guidelines and the experimental protocol number CPCSEA/ IAEC/SPTM/P-32/2013 and CPCSEA/ IAEC/ SPTM/P-10/2013 was approved by Institutional Animal Ethics Committee.
\end{abstract}

\section{Preparation of extract}

The shade dried powdered leaves of $M$ koenigii (200 gm) were defatted with petroleum ether and then exhaustively extracted with $95 \%$ ethanol .The solvent was removed by evaporation using a rotary vacuum evaporator. The extract was subjected to phytochemical screening [12] and acute toxicity studies [13].

The acute toxicity study was performed according to the OECD guidelines 423 in which $2000 \mathrm{mg} / \mathrm{kg}$ of the test extract were administered to the mice orally. All animals were observed within the period of $48 \mathrm{~h}$ for the sign of toxicity. Accordingly, the food intake and body weight were monitored for a period of 14 days.

\section{Efficacy studies}

Hypertension was induced in Wistar albino rats by intra-peritoneal injection of freshly prepared solution of Cadmium chloride $(1 \mathrm{mg} / \mathrm{kg})$ in $0.9 \% \mathrm{w} / \mathrm{v}$ saline, daily for two weeks [14]. The animals were simultaneously given the test extract two weeks before cadmium chloride injection and two weeks along with cadmium chloride injection. At the end of one month the blood pressure was recorded using the invasive blood pressure monitoring system (Iworks data recorder). The animals were divided into eight groups $(n=6)$ :

Group I: Animals receiving normal saline $0.9 \% \mathrm{w} / \mathrm{v}$ for 2 weeks.

Group II: Animals receiving cadmium chloride $(1 \mathrm{mg} / \mathrm{kg})$ for 2 weeks.

Group III: Animals receiving the extract $(150 \mathrm{mg} / \mathrm{kg})$ for two weeks followed by cadmium chloride $(1 \mathrm{mg} / \mathrm{kg})$ for two weeks.

Group IV: Animals receiving the extract $(250 \mathrm{mg} / \mathrm{kg})$ for two weeks followed by cadmium chloride $(1 \mathrm{mg} / \mathrm{kg}$ ) for two weeks.

Group V: Animals receiving Amlodipine $(500 \mu \mathrm{g} / \mathrm{kg})$ for two weeks followed by cadmium chloride $(1 \mathrm{mg} / \mathrm{kg}$ ) for two weeks.
Group VI: Animals receiving Amlodipine $(500 \mu \mathrm{g} / \mathrm{kg})$ for 4 weeks.

Group VII: Animals receiving the extract $(250 \mathrm{mg} / \mathrm{kg})$ for 4 weeks.

Group VIII: Animals receiving the extract $(150 \mathrm{mg} / \mathrm{kg})$ for 4 weeks.

\section{Effect of simultaneous administration of M. koenigii and amlodipine}

To study the effect of combination, four more groups $(n=6)$ were included as follows:

Group I: Animals receiving the extract $(150 \mathrm{mg} / \mathrm{kg})$ and amlodipine $(500 \mu \mathrm{g} / \mathrm{kg})$ for two weeks

Group II: Animals receiving the extract $(150 \mathrm{mg} / \mathrm{kg})$ and amlodipine $(250 \mu \mathrm{g} / \mathrm{kg})$ for two weeks

Group III: Animals receiving the extract $(100 \mathrm{mg} / \mathrm{kg})$ and amlodipine $(500 \mu \mathrm{g} / \mathrm{kg})$ for two weeks

Group IV: Animals receiving the extract $(100 \mathrm{mg} / \mathrm{kg})$ and amlodipine $(250 \mu \mathrm{g} / \mathrm{kg})$ for two weeks

\section{Statistical analysis}

All the data was analysed using one way ANOVA followed by Dunnette's multiple comparison tests.

\section{Results}

The extract shows the presence of steroids, alkaloids, glycosides, flavonoids, phenolic compounds and carbohydrates.

Acute toxicity studies: The animals receiving the extract did not show any mortality, thus it can be said that LD50 of the extract is more than $5 \mathrm{~g} / \mathrm{kg}$.

Effect of administration of M. koenigii: As shown in Figure 1, the decrease in blood pressure in normotensive animals was found to be dose dependent as extract at the dose of $150 \mathrm{mg} / \mathrm{kg}$ and $250 \mathrm{mg} / \mathrm{kg}$ reduced the blood pressure to $94.58 \mathrm{mmHg}$ and $43.87 \mathrm{~mm} \mathrm{Hg}$ respectively. This decrease was found to be significant when compared to normal group, which suggests that the extract has hypotensive effect. Thus, if the extract has to be used in therapy the dose needs to be less than $150 \mathrm{mg} / \mathrm{kg}$. Figure 2 shows that the decrease in blood pressure in hypertensive animals was found to be dose dependent as the extract at the dose of $150 \mathrm{mg} / \mathrm{kg}$ and $250 \mathrm{mg} / \mathrm{kg}$ reduced the blood pressure to $121.85 \mathrm{mmHg}$ and $95.12 \mathrm{~mm} \mathrm{Hg}$ respectively. This decrease was found to be significant in both the groups when compared with hypertensive animals. This shows that the extract has antihypertensive property.

The study proves that ethanolic extract of Murraya koenigii leaf possesses anti-hypertensive effect. The dose optimized is $150 \mathrm{mg} / \mathrm{kg}$ of the extract, since the effect produced is similar to amlodipine administration in hypertensive rats. Moreover, increase in the dose results in hypotensive effect.

Effect of co administration of $M$. koenigii and amlodipine: Mortality was observed in case of groups of rats which received a combination of extract and Amlodipine, wherein Amlodipine was administered orally two hours after administration of the extract. This suggested that there was a potential interaction between the extract and Amlodipine. The mortality occurred even when the dose of 
extract was reduced to $100 \mathrm{mg} / \mathrm{kg}$ and that of Amlodipine to 250 $\mu \mathrm{g} / \mathrm{kg}$.

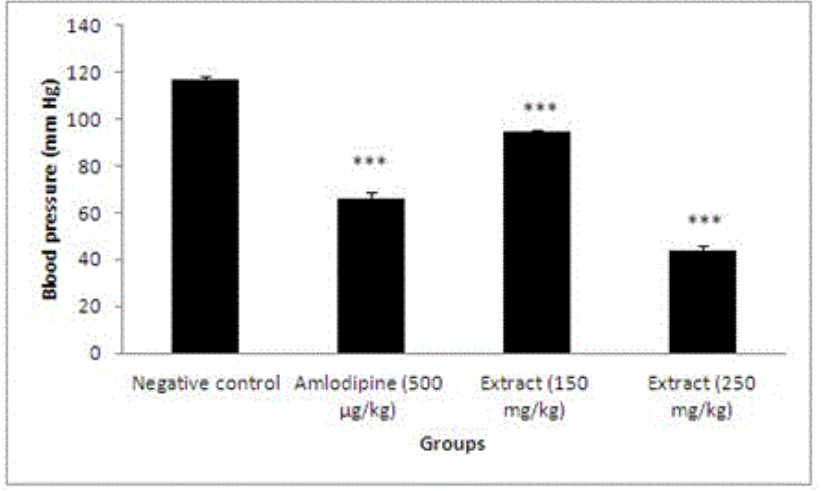

Figure 1: Effect of Murraya koenigii leaf extract at different doses in normotensive groups. Values are mean \pm SEM, ${ }^{* * *} \mathrm{p}<0.001$ when compared with the normal group.



Figure 2: Effect of Murraya koenigii extract at different doses in cadmium chloride induced hypertensive groups. Values are mean \pm SEM, ${ }^{* * *} \mathrm{p}<0.001$ and ${ }^{* *} \mathrm{p}<0.01$ when compared with the hypertensive control group.

\section{Discussion}

The potential interactions between the herbs and the conventional drugs are understood poorly as the research related to them is still in infancy and also because they are significantly under-reported and under-estimated. The drug-herb interactions occur less frequently than predicted making their identification and examination very challenging. It is essential to identify and evaluate the potential drugherb interactions in order to adopt proper safety measures [15]. This is especially true in case of drugs with long elimination half-life (about 33 hours) and those with a narrow therapeutic index, such as Amlodipine.

A number of cases have been reported in the literature where herbal medicines, used for a number of years with safety, suddenly appear to be unsafe, and till date there has been no satisfactory explanation for these adverse effects. Therefore, to confirm the safety of the drugs and plant products for the human use, pharmacological evaluation was performed to predict any interactions and to develop sufficient evidence for selecting a safe dose of the drug in humans.

The group of animals receiving $M$. koenigii extract showed an antihypertensive effect in hypertensive rat model. The model used for the study was cadmium chloride induced hypertension, which causes the mobilization of calcium ions through the $\mathrm{Ca}^{2+}$ ion channel. Since the extract shows an antihypertensive effect in this particular model, it might be altering the movement of calcium ions. However, further investigations to understand the underlying mechanism and to find the phyto-constituent responsible for this effect is required.

The group of rats treated with Amlodipine showed a remarkable decrease in the blood pressure almost similar to the normal group. This can be attributed to the fact that Amlodipine is a well-known calcium channel blocker which inhibits the $\mathrm{Ca}^{2+}$ ion channels thereby inhibiting the trans-membrane influx of calcium ions. This leads to vasodilation of the vascular smooth muscles, decrease in the total peripheral resistance and a subsequent decrease in the blood pressure. However, the co-administration of $M$. koenigii extract and amlodipine resulted in mortality of animals even at reduced dose levels.

As the extract itself was found to possess strong hypotensive action in normal group and anti-hypertensive effect in cadmium chloride induced hypertensive rats, its combination with Amlodipine produced a profound hypotensive effect. This is in accordance with the similar other studies involving interactions of herbs in combination with Amlodipine [11]. A significant diuresis was also observed in animals suggesting excessive loss of sodium and potassium ions. This could have further precipitated hypotension. However to know the exact mechanism of this interaction which possibly may be due to enhanced diuresis, the study needs to be conducted in hypertensive animals.

The study thus suggested that Murraya koenigii extract shows interaction with Amlodipine upon co-administration and the combination results in mortality of animals. There could also be a necessity to investigate the existence of such a pharmaco-dynamic interaction of the extract with other anti-hypertensive drugs to elucidate their exact mechanism. Early identification of such interactions and the mechanism involved therein could be an effective way to avoid the consequences of interactions; provided this identification stage is incorporated early into stages of development of synthetic drugs and herbal medicines. Such studies will also help in educating professionals like doctors, nurses and pharmacists engaged in patient care of possible herb drug interactions and help in avoiding such incidents. Thus investigation of the potential drug-herb interactions should be done and the research must go beyond not only focusing on pharmacological efficacy of botanicals but also in studies that improve their effectiveness in order for humanity to fully benefit from their inherent therapeutic potentials.

\section{Acknowledgement}

The authors are thankful to SPP-SPTM, SVKM's NMIMS for funding the research.

\section{References}

1. Kumar VS, Sharma A, Tiwari R, Kumar S (1999) Murraya koenigii: A review. J of Med and Aromat Plant Sci 21: 1139-1144.

2. Rakesh K Sindhu, Sandeep Arora (2012) Phytochemical and Pharmacognostical Studies on Murraya koenigii L spreng Roots. Drug Invention Today 4: 325-333. 
Citation: Kalyani HB, Ginpreet K, Aadesh K, Saloni D (2015) The Effect of Murraya koenigii Extract on Therapeutic Efficacy of Amlodipine in Rats: Possible Drug-Herb Interaction. General Med 3: 1000153. doi:10.4172/2327-5146.1000153

Page 4 of 4

3. Prashant Tiwari, Bimlesh kumar, Mandeep Kaur et al. (2011) Phytochemical screening and extraction: A review. Int PharmaSci 1: 1.

4. Narasimhan NS, Paradkar MV, Chitguppi VP, Kelkar SL (1975 Alkaloids of Murraya koenigii: Structures of mahanimbine, koenimbine, mahanine, koenine, koenigine, koenidine \& isomahanimbine. Ind J of Chem 13: 993-999.

5. Kureel SP, Kapil RS, Popli SP (1969) Terpenoid alkaloids from Murraya koenigii Spreng. II. The constitution of cyclomahanimbine, bicyclomahanibine, and mahanimbidine. Tetrahedron Lett: 3857-3862.

6. Mhaskar KS, Blatter E, Caius JF (2000) Kirtikar and Basu's Illustrated Indian Medicinal Plants (3rd Edn) Ind Med Sci Ser.

7. Harish Khandral, Anup Pandith and Shruthi SD (2012) A review on Murraya koenigii: multipotential medicinal plant. Asian Journal of Pharmaceutical and Clinical Research 5: 5-14.

8. Gupta P, Nahata A, Dixit VK (2011) An update on Murraya koenigii spreng: a multifunctional Ayurvedic herb. Zhong Xi Yi Jie He Xue Bao 9: 824-833.
9. Bhandari PR (2012) Curry leaf or cure leaf: review of its curative properties. Journal of medical nutrition and nutraceuticals 1: 92-97.

10. Brunton L, Parker K, Blumenthal D, Buxton I (2008) Goodman and Gillman's manual of pharmacology and therapeutics (6th Edn), Mc Graw Hill, United States.

11. Bush TM, Rayburn KS, Holloway SW (2007) Adverse interactions between herbal and dietary substances and prescription medications: A clinical survey.Alternative Therapies 13: 30-35.

12. Khandelwal KR (2006) Practical Pharmacognosy. (16th Edn), Nirali Prakashan, Pune,Maharashtra, India.

13. OECD guideline for testing of chemicals (423) - acute oral toxicity - acute toxic class method 4: 6-13.

14. Balaraman R, Gulati OD, Bhatt JD, Rathod SP, Hemavathi KG (1989) Cadmium-induced hypertension in rats. Pharmacology 38: 226-234.

15. Spinella M (2002) the importance of pharmacological synergy in psychoactive herbal medicines. Altern Med Rev 7: 130-137. 